\title{
PRACTICAL COMPUTATION METHOD FOR LATERAL STABILITY OF THROUGH ARCH BRIDGE
}

\author{
Chen Kong-liang $^{1} \quad$ Wang Rong-hui $^{2} \quad$ Cai Lu-rong ${ }^{3}$
}

${ }^{1}$ Chen Kongliang, Doctor, School of Civil Engineering and Transportation, South China University of Technology. ( ckongliang@163.com.)

${ }^{2}$ Wang Ronghui, Professor, School of Civil Engineering and Transportation, South China University of Technology.

${ }^{3}$ Cai Lurong, Docror, School of Civil Engineering and Transportation, South China University of Technology.

\begin{abstract}
Based on energy principle, an analytical formula for lateral flexible stability capacity of single-rib and double-rib tied through arch bridge is presented under conservative forces and non-conservative forces. The correctness of the analytical formula is verified by two examples of finite element numerical solution, and some important structural parameters of lateral stability capacity are analyzed according to analytical formulas. The conclusions illustrate that increasing the lateral stiffness of arch rib is the most significant way for improvement of lateral stability capacity, and improving the stiffness of traverse brace and lateral stiffness of girder are conducive to greatly improvement of lateral stability capacity, and the lateral stability capacity of arch bridge with traverse brace is about $30 \%$ greater than that of arch bridge without traverse brace.
\end{abstract}

Keywords:tied arch bridge; stability; capacity; non-conservative forces

\section{INTRODUCTION}

The stability ${ }^{[1-7]}$ is an important indicator of carrying capacity for arch bridges. The lateral stability ${ }^{[8-11]}$ of arch bridge is more prominent problem as the bridge span increases. At present, the research of lateral stability about arch bridge is usually taken by the radial loads on circular arch in order to obtain analytical solution, the parabolic and catenary arches are mostly for spreadsheets ${ }^{[12-14]}$. Furthermore, circular arch on small span ratio has a good approximation with the parabolic and catenary arches. Therefore, the lateral stability of circular arch is feasible in practical application instead of the parabolic and catenary arches. Based on some assumptions, an approximate analytical solution for the lateral stability is derived by dual-energy method and some examples are given to verify the correctness of the formula, and some important structural parameters of lateral stability are discussed according to the formula, and some effective ways are proposed to improve calculation for the lateral stability of arch and provid a reference. 


\section{THEORETICAL ANALYSIS}

With regard to the lateral bucking of through tie arch bridge, the method is derived based on the following assumptions:

(1) The arch axis is arc-shaped;

(2) The arch springer is embedded solid boundary conditions;

(3) The axial strain energy is ignored when the arch rib is in the state of lateral bucking;

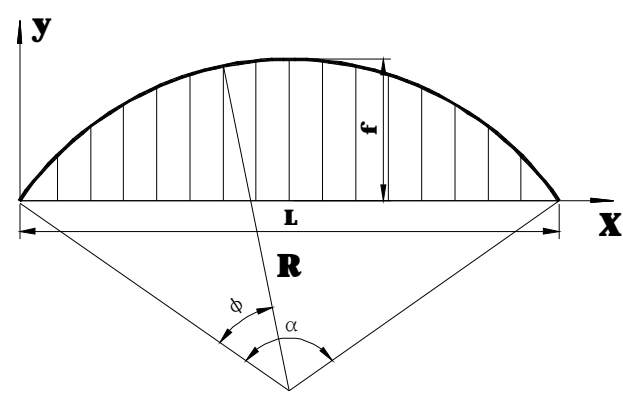

Figure 1 Elevation coordinates

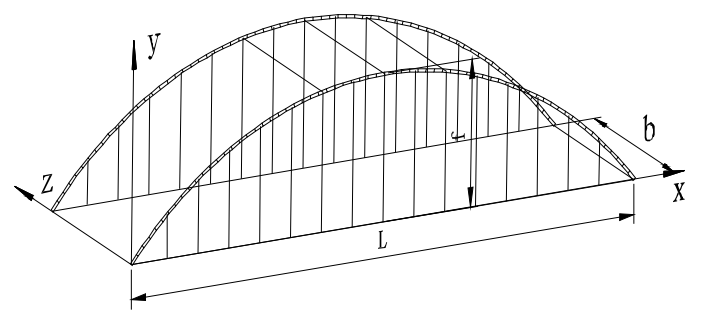

Figure 2 Three-dimensional coordinates

Based on the lateral bucking state of through tied arch bridge, the structural deformation parameters are shown in Figure 1 Figure 2. The dead load of bridge floor system is "q", and $" \alpha "$ is the central angle for the arch rib, and " $l " 、 " \lambda "$ are respectively the span of arch and vector high of arch rib.

By deriving analytical formula in application of Ritz method, the displacement functions of the lateral bucking state (torsion angle $\theta$, lateral displacement $\mathrm{u}$ ) are shown as follows:

$$
\begin{aligned}
& \theta=C_{1}\left(1-\cos \frac{2 \pi \varphi}{\alpha}\right) \\
& u=C_{2}\left(1-\cos \frac{2 \pi \varphi}{\alpha}\right)
\end{aligned}
$$

The above functions are satisfied with solid boundary conditions of bending and torsion, where $\varphi=0$ or $\alpha, \theta=0, \theta^{\prime}=0, u=0, u^{\prime}=0$.

\subsection{The energy of lateral buckling}

The total potential energy are including the following parts when the arch rib is in the state of lateral bucking: the lateral bending strain energy of arch rib; the torsion strain energy of arch rib; the lateral bending strain energy of bridge deck; the external potential energy of 
boom; the potential energy of non-conservative forces of boom; the local deformation potential of arch rib and traverse brace.

(1) Lateral bending strain energy of arch rib

$$
\begin{aligned}
U_{1} & =\frac{1}{2} \int_{s} E I_{y}\left(\frac{\theta}{R}-\frac{d^{2} u}{d s^{2}}\right) d s \\
& =\frac{1}{2} E I_{y} \int_{0}^{\alpha}\left(\frac{\theta}{R}-\frac{1}{R^{2}} \frac{d^{2} u}{d \varphi^{2}}\right)^{2} R d \varphi \\
& =\frac{E I_{y}}{2 R^{3}}\left\{\alpha\left(C_{1} R\right)^{2}+\left(R C_{1}+\beta^{2} C_{2}\right)^{2}\right\}
\end{aligned}
$$

Where: $E I_{y}$ is the lateral bending stiffness of single rib, and $\beta=2 \pi / \alpha$;

(2) Torsion strain energy of arch rib

$$
\begin{aligned}
U_{2} & =\frac{1}{2} \int_{s} G J\left(\frac{d \theta}{d s}+\frac{1}{R} \frac{d u}{d s}\right)^{2} d s \\
& =\frac{G J}{2} \int_{0}^{\alpha}\left(\frac{1}{R} \frac{d \theta}{d \varphi}+\frac{1}{R^{2}} \frac{d u}{d \varphi}\right)^{2} R d \theta \\
& =\frac{G J}{2} \beta^{2}\left(C_{1} R+C_{2}\right)^{2} \frac{\alpha}{2}
\end{aligned}
$$

Where: $G J$ is the torsion stiffness of single rib

(3) The lateral bending strain energy of bridge deck system;

Where: $\xi=u_{D} / u$, and $u_{D}$ is displacement of the bridge deck system, and $E_{D} I_{D}$ is lateral bending stiffness of the bridge deck system.

$$
\begin{aligned}
U_{3} & =\frac{1}{2} E_{D} I_{D} \int_{s}\left[\left(u-u_{D}\right)^{\prime \prime}\right]^{2} d s \\
& =\frac{1}{2} E_{D} I_{D} \int_{0}^{\alpha}\left[\left(u-u_{D}\right)^{\prime \prime}\right]^{2} d \alpha \\
& =\frac{1}{2} E_{D} I_{D}(1-\xi)^{2} C_{2}^{2} \beta^{4} \frac{\alpha}{2}
\end{aligned}
$$

(4) Vertical bending strain energy of traverse brace

$$
\begin{aligned}
U_{4} & =\int_{s} \frac{3 E I_{b x}}{b d} \theta^{2} d s \\
& =\int_{s} \frac{3 E I_{b x}}{b d} \theta^{2} R d \varphi \\
& =\frac{3 E I_{b x}}{b d} C_{1}^{2} R \frac{3 \alpha}{2}
\end{aligned}
$$

Where: $E I_{b x}$ is the vertical stiffness of single rib

(5) Local deformation potential energy of arch and horizontal bending strain energy of traverse brace. 


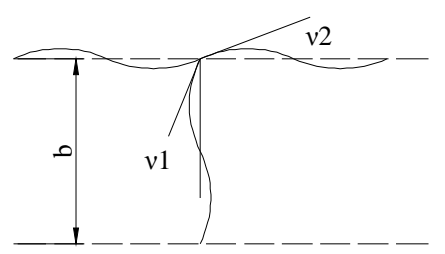

Figure 3 Horizontal deformation of traverse brace

The total energy for local deformation potential energy of arch rib and horizontal bending strain energy of traverse brace is shown as follows:

$$
\begin{aligned}
& U_{5}=\frac{6 E I_{b z}}{b d} \int_{s} \gamma_{1}^{2} d s+\frac{12 E I_{y}}{d^{2}} \int_{s} \gamma_{2}^{2} d s \\
&=\frac{6 E I_{b z}}{b d} \eta \int_{s} \gamma^{2} d s \\
&=\frac{6 E I_{b z}}{b d} \eta \int_{s}\left(\frac{d u}{d s}\right)^{2} d s \\
&=\frac{6 E I_{b z}}{b d} \eta \int_{o}^{\alpha} \frac{1}{R^{2}}\left(\frac{d u}{d t}\right)^{2} R d t \\
&=\frac{3 E I_{b z}}{b d R} \eta \beta^{2} C_{2}^{2} \alpha \\
& \gamma=\gamma_{1}+\gamma_{2} \\
& \eta=\frac{\gamma_{1}}{\gamma}=\frac{1}{1+\gamma_{2} / \gamma_{1}}=\frac{1}{1+d I_{b z} / 2 b I_{y}} \\
& \eta^{\prime}=\frac{\gamma_{2}}{\gamma}=\frac{1}{1+\gamma_{1} / \gamma_{2}}=\frac{1}{1+2 b I_{y} / d I_{b z}} \\
& \frac{6 E I_{b z}}{d^{2}} \gamma_{2}^{2}=\frac{3 E I_{b z}}{h d} \cdot \frac{2 I_{z} h}{I_{b z}} \gamma_{2}^{2}=\frac{3 E I_{b z}}{b d} \gamma_{1} \gamma_{2}
\end{aligned}
$$

Where: $E I_{b z}$ is the traverse stiffness of single rib

(6) The external potential energy of boom

When the arch rib is in the state of lateral bucking, the external potential energy of boom is shown as follows:

$$
\begin{aligned}
V_{1} & =-\frac{q}{2} \int_{s}\left(\frac{d u}{d s}\right)^{2} d s \\
& =-\frac{q}{2} \int_{0}^{\alpha}\left(\frac{d u}{d \varphi}\right)^{2} d \varphi \\
& =-\frac{q}{2} \beta^{2} C_{2}^{2} \frac{\alpha}{2}
\end{aligned}
$$

(7) The potential energy of non-conservative forces of boom

When the arch rib is in the state of lateral bucking, the non-conservative forces potential energy of boom is shown as follows: 


$$
\begin{aligned}
V_{2} & =\frac{q}{2} \int_{s} \frac{u^{2}}{R \cos \left(\frac{\alpha}{2}-\varphi\right)-R+f} d s \\
& =\frac{q}{2} \int_{0}^{\alpha} \frac{u^{2}}{R \cos \left(\frac{\alpha}{2}-\varphi\right)-R+f} R d \varphi \\
& =\frac{q}{2} C_{2}^{2} D
\end{aligned}
$$

$$
\text { Where } D=\int_{0}^{\alpha} \frac{(1-\cos \beta)^{2}}{\cos \left(\frac{\alpha}{2}-\varphi\right)-1+f / R} d \varphi
$$

\subsection{The formula of lateral stability under conservative forces}

According to the concept of Ritz method, the total potential energy of arch under conservative forces could get the extreme data.

$$
\begin{aligned}
& \Pi=U_{1}+U_{2}+U_{4}+U_{5}+V_{1} \quad \frac{\partial \Pi}{\partial C_{i}}=0(i=1,2) \\
& \mu_{1}=\frac{E I_{y}}{G J} \quad \mu_{2}=\frac{E I_{b y}}{G J} \mu_{3}=\frac{E I_{b z}}{G J} \quad n_{1}=\frac{\alpha R}{b} \quad n_{2}=\frac{\alpha R}{d} \\
& \left|\begin{array}{l}
3+\mu_{1} \beta^{2}+\frac{18}{\alpha^{2}} \mu_{2} n_{1} n_{2} \\
\beta^{2}+\mu_{1} \beta^{2} \\
1+\mu_{1} \quad \beta^{2}+\mu-\frac{q R^{3}}{E I_{z}}+\frac{6}{\alpha^{2}} \mu_{3} \eta n_{1} n_{2}
\end{array}\right|\left\{\begin{array}{l}
C_{1} \\
C_{2}
\end{array}\right\}=0
\end{aligned}
$$

When the determinant is zero, it can get a critical bucking load in the state of lateral bucking. According to the geometric relationship of Figure 1: $R=\frac{1}{8 \lambda}+\frac{\lambda}{2}$, then we have

$$
\begin{gathered}
Q_{c r}=\frac{\left[\begin{array}{l}
3 \mu_{1}+2 \beta^{2}+\mu_{1} \beta^{4}-2 \mu_{1} \beta^{2}+\frac{18}{\alpha^{2}} \mu_{3} \eta n_{1} n_{2}+\frac{6}{\alpha^{2}} \eta \mu_{1} \mu_{3} n_{1} n_{2} \beta^{2} \\
+\frac{18}{\alpha^{2}} \mu_{2} n_{1} n_{2} \beta^{2}+\frac{18}{\alpha^{2}} \mu_{1} \mu_{2} n_{1} n_{2}+\frac{108}{\alpha^{4}} \mu_{2} \mu_{3} \eta n_{1}^{2} n_{2}^{2}
\end{array}\right]}{\left(3+\mu_{1} \beta^{2}+\frac{18}{\alpha^{2}} \mu_{2} n_{1} n_{2}\right)\left(\frac{1}{8 \lambda}+\frac{\lambda}{2}\right)^{3}} \frac{E I_{y}}{l^{3}} \\
K=\frac{\left[\begin{array}{l}
3 \mu_{1}+2 \beta^{2}+\mu_{1} \beta^{4}-2 \mu_{1} \beta^{2}+\frac{18}{\alpha^{2}} \mu_{3} \eta n_{1} n_{2}+\frac{6}{\alpha^{2}} \eta \mu_{1} \mu_{3} n_{1} n_{2} \beta^{2} \\
+\frac{18}{\alpha^{2}} \mu_{2} n_{1} n_{2} \beta^{2}+\frac{18}{\alpha^{2}} \mu_{1} \mu_{2} n_{1} n_{2}+\frac{108}{\alpha^{4}} \mu_{2} \mu_{3} \eta n_{1}^{2} n_{2}^{2}
\end{array}\right.}{\left(3+\mu_{1} \beta^{2}+\frac{18}{\alpha^{2}} \mu_{2} n_{1} n_{2}\right)\left(\frac{1}{8 \lambda}+\frac{\lambda}{2}\right)^{3}} \quad Q_{c r}=K \frac{E I_{y}}{l^{3}}
\end{gathered}
$$


When the $\mu_{2}, \mu_{3}, n_{1}, n_{2}$ are zero,the critical bucking loads of single-rib arch can be introduced under conservative forces.

$$
\begin{gathered}
Q_{c r}=\frac{\left[3 \mu_{1}+2 \beta^{2}+\mu_{1} \beta^{4}-2 \mu_{1} \beta^{2}\right]}{\left(3+\mu_{1} \beta^{2}\right)\left(\frac{1}{8 \lambda}+\frac{\lambda}{2}\right)^{3}} \frac{E I_{y}}{l^{3}} \quad Q_{c r}=K \frac{E I_{y}}{l^{3}} \\
K=\frac{\left[3 \mu_{1}+2 \beta^{2}+\mu_{1} \beta^{4}-2 \mu_{1} \beta^{2}\right]}{\left(3+\mu_{1} \beta^{2}\right)\left(\frac{1}{8 \lambda}+\frac{\lambda}{2}\right)^{3}}
\end{gathered}
$$

\subsection{The formula of lateral stability under non-conservative forces}

The energy of non-conservative forces is shown as follows:

$$
\begin{gathered}
\Pi=U_{1}+U_{2}+U_{3}+U_{4}+U_{5}+V_{1}+V_{2} \quad \frac{\partial \Pi}{\partial C_{i}}=0 \quad(i=1,2) \quad m=E_{D} I_{D} / G J \\
\left|\begin{array}{cc}
3+\mu \beta^{2}+\frac{18}{\alpha^{2}} \mu_{2} n_{1} n_{2} & \beta^{2}+\mu \beta^{2} \\
1+\mu & \beta^{2}+\mu+\frac{q}{A}(D-1)+m \xi^{2} \beta^{2}+6 \mu_{3} \eta_{1} n_{1} n_{2}
\end{array}\right|\left\{\begin{array}{l}
C_{1} \\
C_{2}
\end{array}\right\}=0
\end{gathered}
$$

Simplify the above determinant, it can get

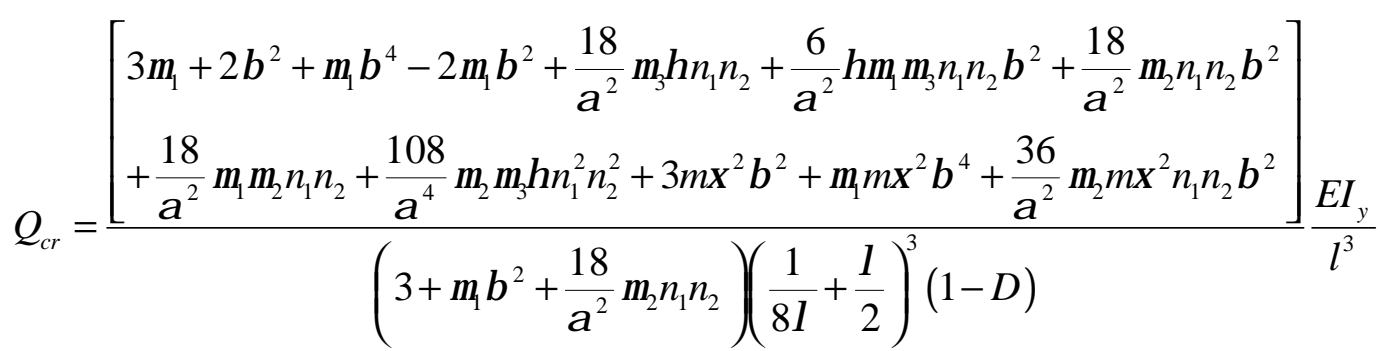

$$
\begin{aligned}
& \text { where } D=3\left(\frac{1}{8 \lambda^{2}}+\frac{1}{2}\right) / \beta^{2} \text {, then we have } \\
& K=\frac{\left[\begin{array}{l}
3 \mu_{1}+2 \beta^{2}+\mu_{1} \beta^{4}-2 \mu_{1} \beta^{2}+\frac{18}{\alpha^{2}} \mu_{3} \eta n_{1} n_{2}+\frac{6}{\alpha^{2}} \eta \mu_{1} \mu_{3} n_{1} n_{2} \beta^{2}+\frac{18}{\alpha^{2}} \mu_{2} n_{1} n_{2} \beta^{2} \\
+\frac{18}{\alpha^{2}} \mu_{1} \mu_{2} n_{1} n_{2}+\frac{108}{\alpha^{4}} \mu_{2} \mu_{3} \eta n_{1}^{2} n_{2}^{2}+3 m \xi^{2} \beta^{2}+\mu_{1} m \xi^{2} \beta^{4}+\frac{36}{\alpha^{2}} \mu_{2} m \xi^{2} n_{1} n_{2} \beta^{2}
\end{array}\right]}{\left(3+\mu_{1} \beta^{2}+\frac{18}{\alpha^{2}} \mu_{2} n_{1} n_{2}\right)\left(\frac{1}{8 \lambda}+\frac{\lambda}{2}\right)^{3}(1-D)} Q_{c r}=K \frac{E I_{y}}{l^{3}}
\end{aligned}
$$

When the $\mu_{2}, \mu_{3}, n_{1}, n_{2}$ are zero, the lateral stability capacity of single-rib arch can be introduced under non-conservative forces.

$$
Q_{c r}=\frac{\left[3 \mu_{1}+2 \beta^{2}+\mu_{1} \beta^{4}-2 \mu_{1} \beta^{2}+3 m \xi^{2} \beta^{2}+\mu_{1} m \xi^{2} \beta^{4}\right]}{\left(3+\mu_{1} \beta^{2}\right)\left(\frac{1}{8 \lambda}+\frac{\lambda}{2}\right)^{3}(1-D)} \frac{E I_{z}}{l^{3}} \quad Q_{c r}=K \frac{E I_{y}}{l^{3}}
$$




$$
K=\frac{\left[3 \mu_{1}+2 \beta^{2}+\mu_{1} \beta^{4}-2 \mu_{1} \beta^{2}+3 m \xi^{2} \beta^{2}+\mu_{1} m \xi^{2} \beta^{4}\right]}{\left(3+\mu_{1} \beta^{2}\right)\left(\frac{1}{8 \lambda}+\frac{\lambda}{2}\right)^{3}(1-D)}
$$

\section{EXAMPLE}

\subsection{The example of single-rib arch}

The first example is single-rib parabolic tied arch bridge, with a $120 \mathrm{~m}$ span length and $11.2 \mathrm{~m}$ width.The span ratio of arch is $1 / 4.6$, and the section size of steel-box rib is $1.8 \times 2 \mathrm{~m}$. The tie is low-slack and high-strength galvanized steel wire, and the boom is high-strength parallel steel wire.

For simplicity, the parabolic arch is approximated with a circular arch, the basic parameters are as follows: $l=120 \mathrm{~m}, \lambda=1 / 4.6, \quad R=82 \mathrm{~m}, \quad \alpha=1.64 \mathrm{rad}, \quad E I_{y}=3.83 \times 107 \mathrm{kNm} 4$, $G J=1.79 \times 107 \mathrm{kNm} 4$; and then $\mu 1=0.48, \mathrm{~m}=46.2, \beta=3.82$. According to formula (12) and (17), the analytical lateral stability capacity under conservative and non-conservative forces are respectively $Q_{c r}=948 \mathrm{kN} / \mathrm{m}$ and $Q_{c r}=2210 \mathrm{kN} / \mathrm{m}$. The spatial bridge model is build up by Ansys, imposing $116 \mathrm{kN} / \mathrm{m}$ on the deck, the buckling mode are respectively 7.8 and 20.6 under conservative forces and non-conservative forces. Therefore, the calculated lateral stability capacity under conservative forces and non-conservative forces are respectively: $116 \times 7.8=904.8 \mathrm{kN} / \mathrm{m}, 116 \times 20.6=2389.6 \mathrm{kN} / \mathrm{m}$; the results in recommended analytical formula are difference of $4.0 \%, 7.5 \%$ with the calculated results.

\subsection{The example of double-rib arch}

The second example is a simply supported double-rib parabolic tied arch bridge, with a span $62 \mathrm{~m}$ length and $15 \mathrm{~m}$ the width. The span ratio of arch is $1 / 5$. The arch is steel tube which is of $1.1 \mathrm{~m}$ diameter; and the tie is the low-slack and high-strength galvanized steel wire, and the boom is high-strength parallel steel wire with three $\phi 600 \times 12 \mathrm{~mm}$ traverse brace in arch.

For simplicity, the parabolic arch is approximated with a circular arch, the basic parameters are as follows: $l=62 \mathrm{~m}, \lambda=1 / 5, R=45 \mathrm{~m}, \alpha=1.52 \mathrm{rad}$, and the distance between two arches is $b=10.5 \mathrm{~m}$, and the distance between traverse braces is

$\mathrm{d}=12.9 \mathrm{~m} ;{ }^{E I_{y}}=0.35 \times 1010 \mathrm{~m} 4, \quad G J=0.12 \times 1010 \mathrm{~m} 4, \quad E I_{b x}, E I_{b z}=2.1 \times 108 \mathrm{~m} 4$; and then $\mu 1=0.33, \mathrm{~m}=56, \beta=4.13, \mu 2, \mu 3=0.06$. According to formula (10) and (12), the analytical lateral stability capacity under conservative and non-conservative forces are respectively 
$Q_{c r}=810 \mathrm{kN} / \mathrm{m}$ and $Q_{c r}=2152 \mathrm{kN} / \mathrm{m}$. The model is build up by Ansys, imposing $35 \mathrm{kN} / \mathrm{m} 2$ on the deck, and the buckling mode are respectively 4.93 and 12.84 under conservative forces and non-conservative forces, and the calculated lateral stability capacity under conservative forces and non-conservativ forces are respectively: $35 \times 5.25 \times 4.93=905.9 \mathrm{kN} / \mathrm{m}$ and $35 \times 5.25 \times 12.84=2359.4 \mathrm{kN} / \mathrm{m}$; the results in recommended analytical formula are difference of $10.6 \%, 8.8 \%$ with the calculated results.

\section{PARAMETER DISCUSSION OF LATERAL STABILITY CAPACITY}

According to the formula (12) and (17), the lateral stability factors of single-rid are invloved in the following parameters: the lateral bending stiffness of arch rib, and the ratio between lateral bending stiffness of arch rib and torsional stiffness of arch rib " $\mu_{1}$, and the span ratio of arch " $\lambda$ "; furthermore, the lateral stability of factors under non-conservative forces should also be considered the ratio between lateral bending stiffness of bridge deck and lateral bending stiffness of arch rib. In formula (10) and (15), besides the factors mentioned above, the factors of double-rid arch bridge should also be considered the ratio " $\mu_{2}, \mu_{3}$ " between the bending stiffness of traverse brace and torsional stiffness of arch rid. In Figure 4 Figure 5, Figure 7 Figure 9, "c" stands for conservative forces, and "non-c" stands for non-conservative forces.

\subsection{Parameter discussion of single-rid lateral stability capacity}

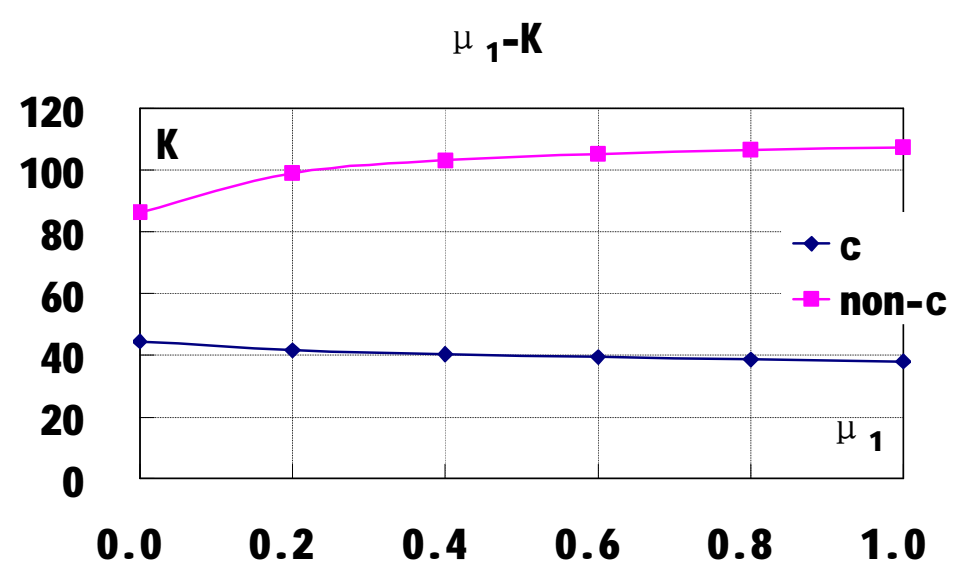

Figure 4 the $\mu_{1}-\mathrm{K}$ curves $(\lambda=0.2, \mathrm{~m}=40)$ 


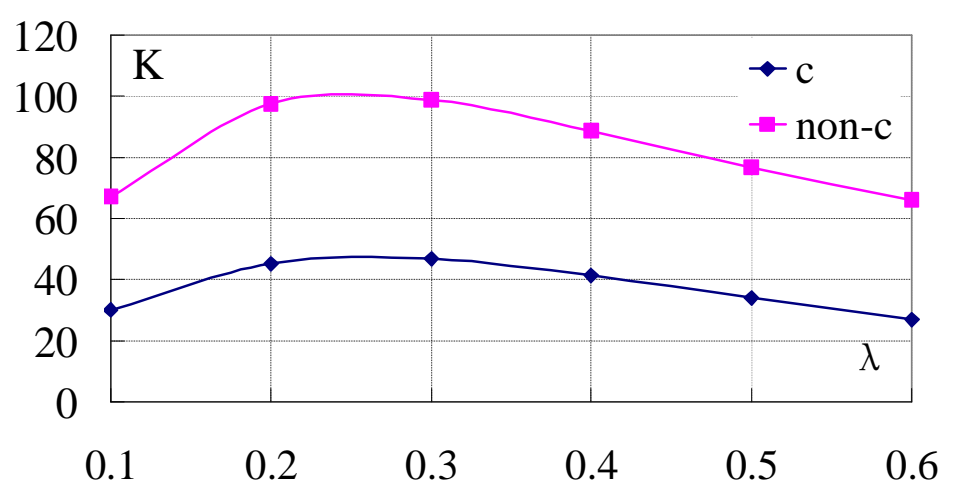

Figure 5 the $\lambda$-K curves $\left(\mu_{1}=0.5, \mathrm{~m}=40\right)$

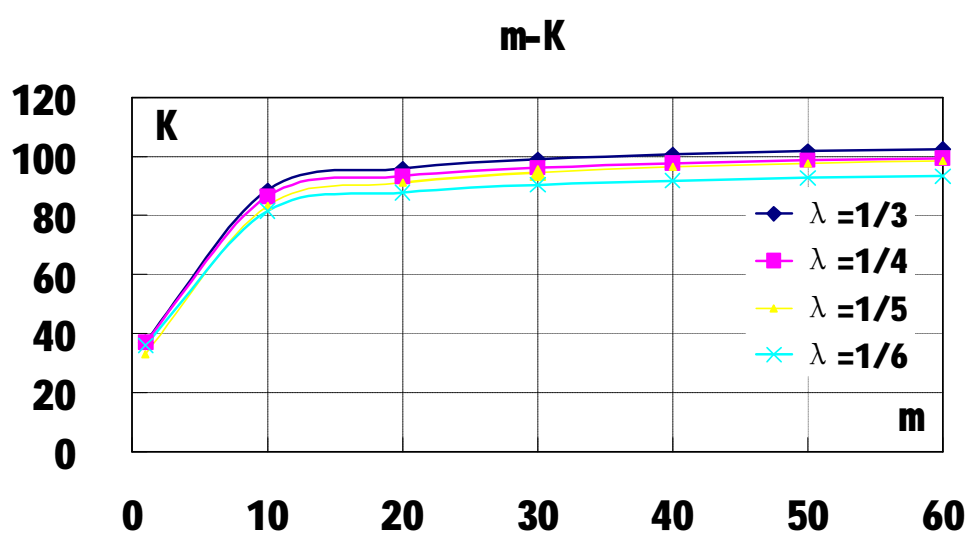

Figure 6 the $\mathrm{m}-\mathrm{K}$ curves $\left(\mu_{1}=0.5, \lambda=0.2\right)$

(1) In formula (12) and (17), the lateral stiffness is the most direct and effective way for lateral stability under conservative forces and non-conservative forces.

(2) As shown in Figure 4 Figure 5, the lateral stability capacity coefficient K under non-conservative forces is much more favorable than this under conservative forces, and the datas show that the lateral stability capacity under non-conservative forces is $2 \sim 3$ times than this under conservative forces.

(3) As shown in Figure 4, the lateral stability capacity coefficient $\mathrm{K}$ decreases $16 \%$ with the increase of ${ }^{\mu_{1}}$ from 0 to 1 under conservative forces; while $\mathrm{K}$ improves $24 \%$ as

$\mu_{1}$ increases from 0 to 1 under non-conservative forces, but $\mu_{1}$ is not obvious effect on the improvement of the lateral stability capacity of arch.

(4) As shown in Figure 5, the trend of the lateral stability capacity coefficient $\mathrm{K}$ is that the curve first increase up to the maximum value and then gradually decrease to stable value with the increase of $\lambda$ under conservative and non-conservative forces. The optimum value of span ratio is between 0.2 and 0.3 ; When the span ratio $\lambda$ is constant, the lateral stability capacity is greater with the lower span.

(5) As shown in Figure 6, the lateral stability capacity improves largely with the increases 
of ratio $\mathrm{m}$ from 0 to 10 times under non-conservative forces; while the lateral stability capacity improves a lesser extent while $\mathrm{m}$ is between 10 to 60 times, and lateral stability capacity of arch is gradually stabilized with the increase of $\mathrm{m}$; but the change of $\lambda$ has little effect on lateral stability capacity with the increase of $\mathrm{m}$.

\subsection{Parameter discussion of double-rid lateral stability capacity}

(1) In formula (10) and (15), the flexural lateral stiffness is the most direct and effective way for lateral stability under conservative forces or non-conservative forces, which is qutie consistent with the single-rid arch bridge.

(2) The lateral stability capacity under non-conservative forces is much more favorable than this under conservative forces, and the data show that the lateral stability capacity under non-conservative forces is $2 \sim 3$ times than this under conservative forces, which is also quite in agreement with the single-rid arch bridge.

(3) As shown in Figure 7, the lateral stability capacity of the arch improves with the increase of $\mu_{1}$ under conservative or non-conservative forces, but coefficient $\mathrm{K}$ increases not more than $1 \%$ when $\mu_{1}$ is increasing between 0 and 1 ; and $\mu_{1}$ is not a susceptible for the lateral stability capacity of arch;

(4) As shown in Figure 8, the regularity for span ratio $\lambda$ on the lateral stability capacity under conservative or non-conservative forces, which is in quite agreement with the single-rid arch bridge.

(4) As shown in Figure 9, the traverse brace is quite important for lateral stability capacity under conservative or non-conservative forces; the lateral stability capacity increases about one times when the ratio $\mu_{2}, \mu_{3}$ are improving from 0.05 to 0.3 .

(5) As shown in Figure 10 , the lateral stability capacity coefficient $\mathrm{K}$ is increasing when $\mathrm{m}$ is between 10 and 60 under non-conservative forces; but changing $\lambda$ has little effect to lateral stability of arch with the increase of $\mathrm{m}$.

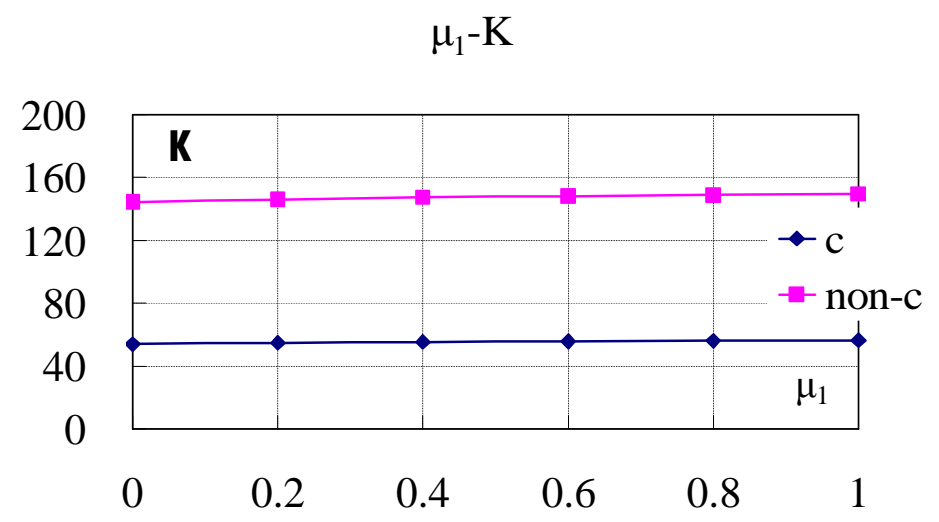

Figure 7 the curve of $\mu_{1}-K\left(\lambda=0.2, \mu_{2}, \mu_{3}=0.05\right)$ 


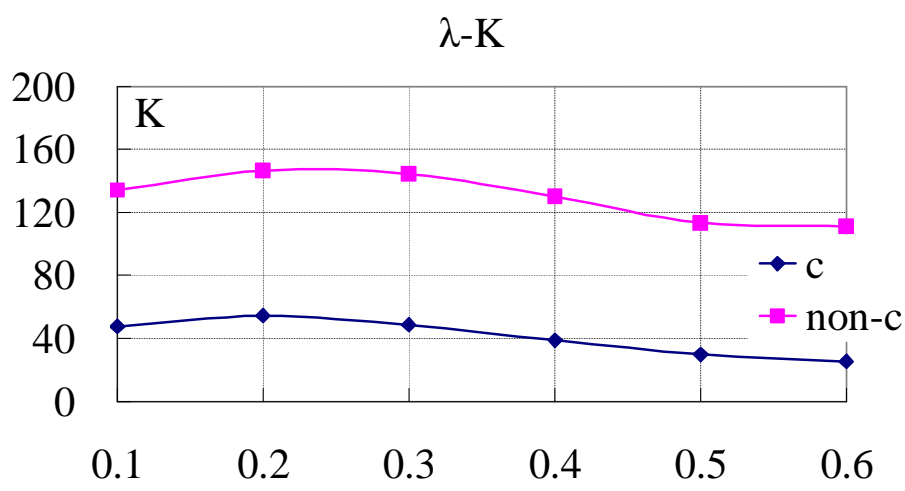

Figure 8 the curve of $\lambda-K\left(\mu_{1}=0.33, \mu_{2}, \mu_{3}=0.05, m=56\right)$

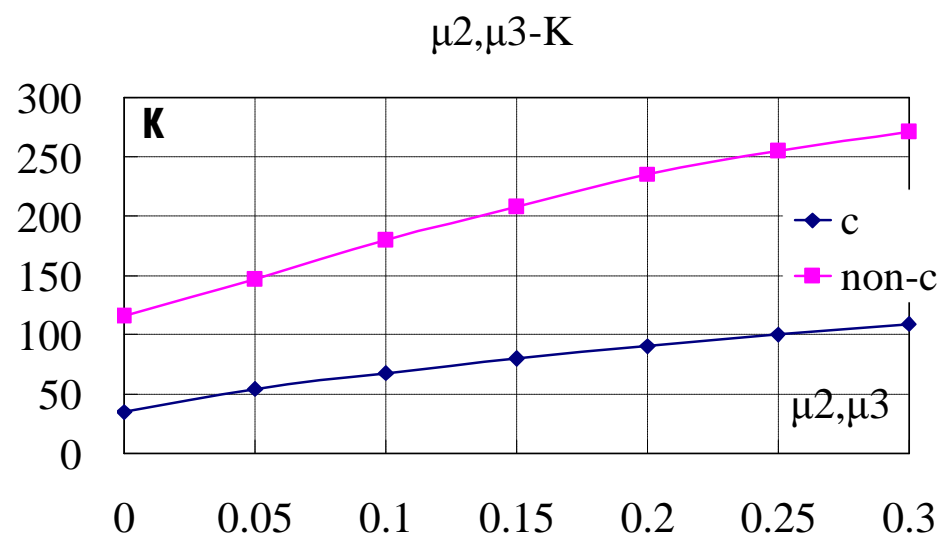

Figure 9 the curve of $\mu 2, \mu 3-\mathrm{K}\left(\mu_{1}=0.33, \lambda=0.2, \mathrm{~m}=56\right)$

$m \cdot K$

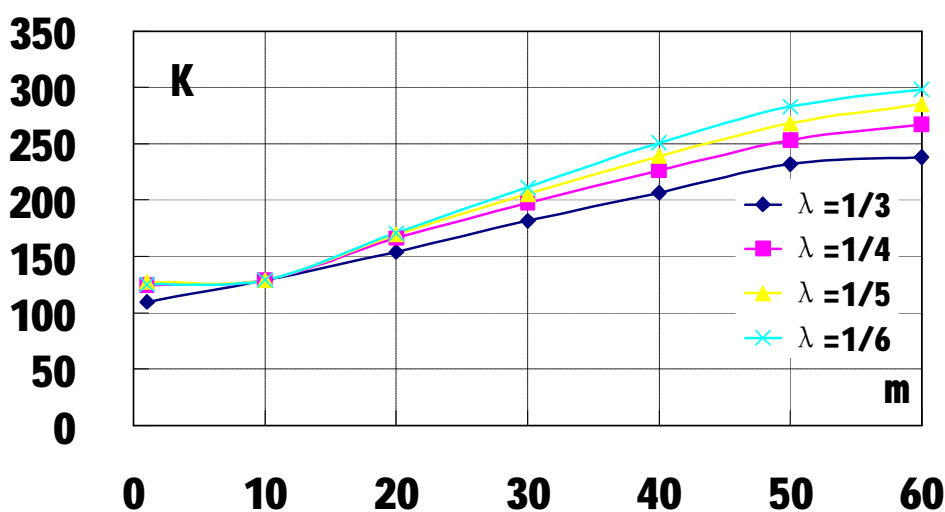

Figure 10 the curve of $\mathrm{m}-\mathrm{K}\left(\mu_{1}=0.33, \mu_{2}, \mu_{3}=0.05\right)$ 


\section{CONCLUSIONS}

As mentioned above, we can draw following conclusions:

(1) The elastic lateral stability capacity analytical formula of arch bridge is a more comprehensive description about the bridge span, the span ratio, the cross-section flexural lateral rigidity ,the lateral torsional stiffness, the deck stiffness and other factors of single- rib and double-rib arch bridge under conservative forces and non-conservative forces for the lateral stability capacity of arch.

(2) The lateral stability capacity under non-conservative forces is much more favorable than this under conservative loads, and the lateral stability capacity under non-conservative forces is 2 3 times than this under conservative forces.

(3) The flexural lateral stiffness of cross-section is the most direct and effective way for lateral stability capacity; while increasing the ratio between lateral bending stiffness of arch rib and torsional stiffness of arch rib is not obvious effect on lateral stability capacity of arch.

(4) The optimum span ratio is between 0.2 to 0.3 , and the lateral stability capacity of arch is gradually stabilized with the increase of the span ratio;

(5) The lateral stiffness of traverse brace and the lateral stiffness of bridge deck are important way for lateral stability capacity of arch, and the lateral stability capacity of arch bridge with traverse brace is about $30 \%$ greater than lateral stability capacity of arch bridge without traverse brace.

\section{REFERENCES}

[1] Xiao Ru-cheng, Sun Hai-Tao, Jia Li-jun. "Kunshan Yufeng Rridge-Design Of The First Long-Span leaning-Type Arch Bridge Without Thrust". China Civil Engineering Journal. Vol. 38, No.1, pp: 78-83. 2005

[2] Xing Fan, Zhu-Bing, Wang Xiao-Ping. "Stability Analysis For CFST Basket Handle Arch Bridge". Proceedings of the 2nd International Conference on Transportation Engineering", ICTE 2009, v345, pp 1378-1383. 2009

[3] Huang li-Ji, Guo Yan Lin. "In-plane Buckling Behavior Of I-sectional Arches With Web Openings". Engineering Mechanics. Vol. 23, No. 3, pp:126-133. 2006

[4] Seung-Eock Kim, Se-Hyu Choi, Sang-Soo Ma. "Performance based design of steel arch bridges using practical inelastic non-linear analysis". Journal of Constructional Steel Research". No. 59. pp: 91-108. 2003

[5] Wang Ji, Zhang Ming-Zhong, Guo Xiao-Li. "Nonlinear Stability Analysis On The Concrete Casting Step Of Long-Span Concrete-Filled Steel Tube Arch Bridge". 6th International Forum on Advanced Material Science and Technology, IFAMST 2008, June 12, 2008-June 14. 2008

[6] Cheng Jin, Li Q.S. "Reliability Analysis Of A Long Span Steel Arch Bridge Against Wind-induced Stability Failure During Construction". Journal of Constructional Steel Research, Vol. 65, No. 3, pp:552-558, March 2009. 2009

[7] Liu Muyu, Gong Kai, Sun Xiangdong. "Dynamic Characteristics Analysis of Single Rib Braces CFST Arch Bridge”. Journal of Wuhan University of Technology. Vol. 33, No. 6, pp:1104-1107. 2009

[8] Bergmeister Konrad, Capsoni Antonio, Corradi Leone. "Lateral elastic stability of slender arches for bridges including deck slenderness". Structural Engineering International: Journal of the International Association for Bridge and Structural 
Engineering.Vol. 19, No.2. pp: 149-154. 2009.

[9] Pan Sheng-Shan, Zhang Zhe, Huang Cai-Liang. "Research on lateral stability influenced by inclined angle of through X-type double ribs arch bridge". Journal of Dalian University Of Technology, Vol., 48, No.6, pp: 845-850. 2008

[10] Jin Bo, Zhao Yue-Yu, Feng Rui. "Effect of lateral brace on the stability of cable-stayed concrete filled steel tube arch bridge". Journal of Hunan University (Natural Sciences), Vol. 33, No.6, pp: 6-10. 2006

[11] Peng Wei, Pan Yong-qing, Du Shi-gui. "Parametric investigation on lateral buckling of concrete filled steel tube arch bridges". Journal Of Zhejiang University Of Technology. Vo. 32, No. 1, pp:6-10. 2004

[12] Tan Hong-Xia, Chen Zheng-Qing, Feng Zhou-Quan. "Practical Computation Method for Out-of-plain Stability Coecients of Arch Structures". Chinese Journal Of Engineering Mathematics.Vol. 27, No.3, pp: 49-502. 2010

[13] A.S.Nazmy. "Stability and load-carrying capacity of three-dimensional long-span steel arch bridges". Computers \& Structures.Vol. 65, No.6, pp:857-868. 1997.

[14] Gianmarco de Felice. "Assessment of the load-carrying capacity of multi-span masonry arch bridges using fibre beam elements". Engineering Structures.Vol. 31, No.8, pp:1634-1647. 2009. 